\title{
CAPÍTULO IV
}

\section{VIGILANCIA Y SEGURIDAD CON PROCESAMIENTO DE IMÁGENES}

\author{
Mario A. Yandar-Lobon
}

Ingeniero de Sistemas. Esp. Gestión de Proyectos. MSc. Matemática Aplicada, Docente Investigador Facultad de Ingeniería. Fundación Universitaria Unipanamericana. Correo electrónico: mayandar@gmail.com. ORCID ID: 0000-0003-1510-266X

\section{Andrés Esteban Puerto Lara}

Ingeniero electrónico. MSc. Docente Facultad de Ingeniería. Fundación Universitaria Panamericana - Unipanamericana. Correo electrónico: aepuertol@unipanamericana.edu.co.

\section{Resumen}

Los conceptos de vigilancia y seguridad se han dado por sentado en las sociedades urbanas colombianas. La vigilancia, desde la perspectiva de la observación implica el conocer lo que pasa, mientras que la seguridad se asocia al cuidado de personas y bienes. Desde el punto de vista del sector en Colombia, ciertamente ha habido un auge creciente, no solo en la demanda de los servicios, sino de tecnología asociada, como cámaras de vigilancia de manera individual y en circuitos cerrados (CCTV), sistemas de alarmas, autenticación biométrica para control de acceso, entre otros. La industria 4.0 supone un impacto relevante en diversos aspectos de una vigilancia y seguridad, principalmente porque el sector demanda servicios $7 \times 24$ con altos niveles de confiabilidad, teniendo hoy por hoy la tendencia a automatizar cada vez más sus operaciones. En la actualidad, este sector registra mucha más información de la que es procesada y retroalimentada eficazmente para la toma de decisiones. El presente análisis tiene como objetivo principal el estudio de la incorporación de técnicas de reconocimiento de imágenes y procesamiento de video en tiempo real para aplicaciones relacionadas con la seguridad perimetral en diferentes lugares que presenten diferentes tipos de actividades. Estas técnicas involucran visión artificial por medio de redes neuronales convolucionales utilizando sistemas de computación paralela que permiten procesar cantidades significativas de imágenes con resultados precisos y en tiempo real que benefician la toma de decisiones en el momento de afrontar un problema de seguridad. Utilizando las técnicas mencionadas se evaluaron videos en algunos sitios públicos y mediante la detección de personas se obtuvieron ciertos parámetros como el flujo de personas. Finalmente, este tipo de parámetros pueden ser utilizados para evaluar la toma de decisiones brindando un apoyo a las empresas de vigilancia en zonas de se presentan altas concurrencias de personas y que pueden tener como consecuencia algunos incidentes de inseguridad.

Palabras clave: circuito cerrado de televisión, Inteligencia Artificial, Redes Neuronales, vigilancia privada, visión artificial, 


\title{
SURVEILLANCE AND SECURITY WITH IMAGE PROCESSING
}

\begin{abstract}
Surveillance and safety concepts are given as a reality in Colombian society. Surveillance, from observation perspective is related with just watching different situations, meanwhile safety is associated with taking care people or possessions. Certainly, in Colombia, this sector has experimented a growth, not just in services demand, also technologies like CCTV cameras, alarms and biometric systems. 4.0 industry supposes a relevant impact in many aspects of surveillance and safety, mainly because this industry demands $7 \times 24$ services with high reliability levels and having intentions of getting involve in automated operations. This sector gets a lot of amounts of data, but this data is stored and not processed properly. This chapter has as main target the study of Artificial intelligence and real time image processing techniques in order to being implemented y surveillance system on public places in Colombia. These techniques include convolutional neural networks and parallel computing methods with the purpose of process big quantities of data in real time with precise results enhancing the making decisions process when a safety incident es presented. Mentioned methods were evaluated with videos taken in some public places. Parameters like the number of persons in certain time (flow of persons) were obtained. Finally, these kinds of parameters can be used to evaluating fast decisions giving a support to surveillance companies in zones where the number of persons is high every day.
\end{abstract}

Keywords: Artificial intelligence, artificial vision, closed circuit television, Neural Networks, private surveillance.

Este capítulo es el resultado de un proyecto de investigación del Grupo de Investigación en Ingenierías GIIS en conjunto con el Grupo EGE titulado "Creación de propuestas de valor para las organizaciones con base en Tecnologías Digitales Emergentes". Finalizado en el año 2019 y financiado por Unipanamericana Compensar Institución Universitaria Bogotá (Colombia).

\section{Introducción}

El sector de la vigilancia privada ha tenido un impacto importante en la economía, no solo su aporte al PIB (Producto interno bruto), sino en el empleo que genera y los servicios que presta a las organizaciones. En los últimos años, la mayor parte de la industria ha tenido una transformación tecnológica importante al incorporar cámaras y otros equipos que permiten mejorar los procesos a realizar.

Por otro lado, la inseguridad es una constante en todo el mundo y en algunos lugares tanto las fuerzas privadas como públicas están perdiendo capacidad ante tantos incidentes de este tipo. Por eso es necesario recurrir a herramientas tecnológicas que proporcionen información oportuna para tomar decisiones rápidas y así evitar incidente de mayor envergadura. 
La Posibilidad de contar con sistemas que analicen datos en tiempos considerablemente inferiores respecto a las capacidades del ser humano como por ejemplo visualizar una situación que requiera analizar muchas horas de video a causa de un evento de inseguridad en donde se vea involucrado alguien con cierta descripción física específica, requiere de un alto esfuerzo cognitivo y probablemente, sea necesario, el apoyo de una o más personas, lo que podría genera aumentos en los costos de logística y retrasaría la toma de decisiones fundamentales en la solución del incidente. En cambio, si se utilizara un sistema basado en inteligencia Artificial que por medio de la información suministrada del sospechoso pueda inspeccionar esas horas de video en cuestión de minutos e identificar inmediatamente a la persona que se ajuste a esas características eleva inmediatamente la capacidad operativa del cuerpo de seguridad.

Esto conlleva, a revisar si los sistemas de seguridad poseen la capacidad suficiente para afrontar situaciones de inseguridad de manera oportuna por medio de las herramientas actuales, además si su análisis y estimación de futuros incidentes contiene la cantidad de datos necesarios para poder afrontarlos (Fuentes y Velastin, 2004).

Es por esto que se plantea como idea principal el estudio del campo de la vigilancia perimetral en Colombia y la posibilidad de incorporar nuevas tecnologías, principalmente aquellas que involucran inteligencia artificial, además evaluar si son eficientes en el momento de tomar decisiones que requieran rápida reacción o de análisis profundos que permitan predecir futuros incidentes.

Este documento presenta en su inicio, las generalidades del sector en Colombia, la identificación de problemática de la industria, así como una estimación del impacto de la tecnología de videovigilancia y su uso por parte de las empresas. Posteriormente, se relacionan algunos elementos de la inteligencia artificial y las pruebas de aplicación en el reconocimiento de personas y objetos en videos.

Finalmente, se relacionan los elementos de la industria y la tecnología de cara a la problemática identificada y las posibilidades de las empresas sobre las promesas de valor en competitividad, productividad y conocimiento.

\section{Marco conceptual}

\section{Vigilancia privada en Colombia}

El concepto de vigilancia, en su concepción más amplia, busca el observar y analizar el entorno. De acuerdo con Pérez y Navarrete, (2018, p. 154), el sector se encuentra:

La última década ha sido testigo de una importante expansión de los servicios de seguridad y vigilancia en América Latina, con una tasa media de crecimiento anual del $8 \%$, casi el doble de la tasa global de expansión de la seguridad privada. De acuerdo con la Superintendencia de Vigilancia y Seguridad Privada, en Colombia en 2016, el sector contaba con cerca de 900 empresas, la mayoría de ellas pequeñas y empleaba a más de 256 mil personas. Sin embargo, dado que en el sector existe una alta informalidad, estas cifras podrían estar subestimadas. En todo caso, se trata de un sector relevante para la economía y dado su objetivo, también para la seguridad ciudadana... 
El mismo estudio señala que durante los últimos cinco años, el sector ha experimentado un crecimiento anual compuesto de $12,18 \%$ en sus ingresos y de $7,6 \%$ en sus utilidades". También se indica que: "El número de servicios prestados durante los últimos 22 años ha aumentado de forma progresiva: de 763 en 1994 pasaron a 6056 en 2017 (ver gráfico 1). A simple vista se evidencian tres etapas en la evolución de los servicios de seguridad y vigilancia en el país: En la primera etapa (1994-2002) hay un aumento drástico en los servicios; en la segunda (2003-2010) se presenta un comportamiento lateral y durante la última etapa (2010-2016) se evidencia nuevamente un incremento en los servicios prestados.

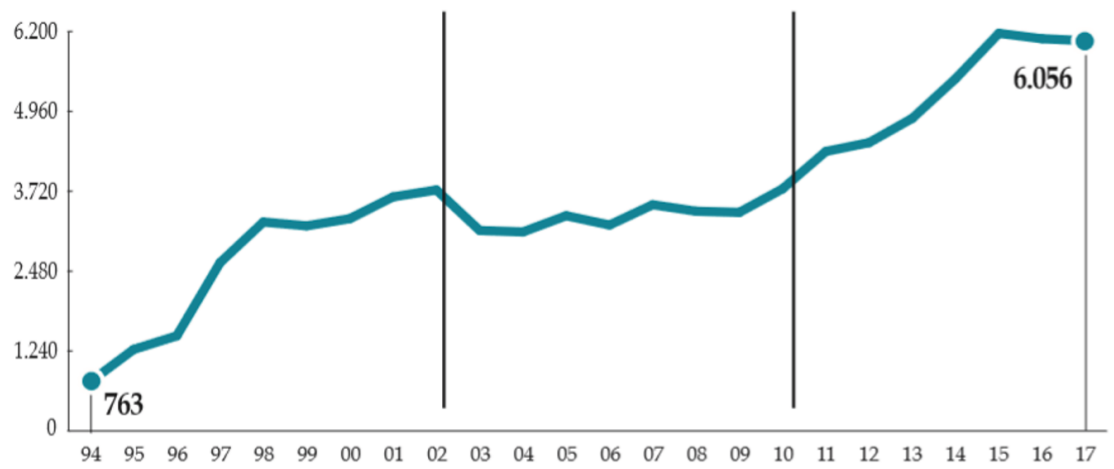

\section{Gráfica 1. Evolución servicios Supervigilancia en Colombia.}

Fuente: Pérez y Navarrete (2018).

La industria de vigilancia y seguridad en las grandes ciudades de Colombia se ha tornado en un sector altamente competitivo. Es en estas ciudades donde masivamente los ciudadanos visitan los establecimientos de comercio en general, de igual forma gran cantidad de oficinas y empresas requieren contar con servicios especializados de seguridad y vigilancia. Uno de los retos de cara al futuro para el sector lo ilustra Pérez y Navarrete (2018), cuando manifiestan que la alta rotación de los empleados se percibe por parte de la mayoría de las empresas como una de las situaciones más problemáticas, siendo en promedio del $25 \%$ al año. Basado en este panorama, el desarrollo de tecnologías emergentes puede proveer propuestas de valor para ayudar en la automatización de procesos, tendientes a optimizar el recurso humano en este sector.

\section{Videovigilancia en Colombia}

La tecnología de videovigilancia se ha popularizado en los últimos años en Colombia y Latinoamérica en general (Security Magazine, 2009). Estos sistemas también denominados Circuitos cerrados de televisión (Closed-circuit television - CCTV). Las distintas tecnologías de registro de imágenes y video han proporcionado mayor eficiencia sobre el accionar de las empresas de vigilancia y seguridad (Bouma et al., 2016). La visualización de las imágenes 
con cámaras situadas en puntos estratégicos de las zonas a vigilar, proporcionan puntos de vista privilegiados para el análisis continuo de lo que ocurre (Lio, 2015).

A su vez, la posibilidad de almacenar estas imágenes, proporcionan material invaluable a la hora de realizar un proceso forense de actividades criminales. Por el tipo de información registrada, que es principalmente video, los sistemas de almacenamiento frecuentemente se llenan por la abundante información registrada. Realizar procesos de búsqueda o generar reacción inmediata a partir del material de video, muchas veces es una tarea que toma bastante tiempo (Keval, 2008). En Colombia, el uso de estos sistemas se encuentra regulado por la Ley de La protección de los datos personales (Ministerio de Comercio, Industria y Turismo, 2012).

Particularmente la videovigilancia ha tenido un impacto relevante sobre la seguridad en centros comerciales, con una amplia expansión en los últimos años con el crecimiento de los centros poblados (Chen, Chiang, y Storey, 2013). La seguridad ha sido uno de los elementos motivadores para los consumidores como un elemento de vital importancia a la hora de elegir dónde comprar (George, 2018). Algunas ventajas citadas para estos sistemas son:

- Amenazas delictivas (vandalismo, robo).

- Peleas o discusiones.

- Situaciones de emergencia.

- Niños perdidos.

Adicionalmente a lo mencionado, el tipo de imágenes registradas por estos sistemas, como se muestra en la ilustración 1, también puede utilizarse para identificar afluencia de personas (Khan, 2016), identificar elementos sospechosos, entre otros.

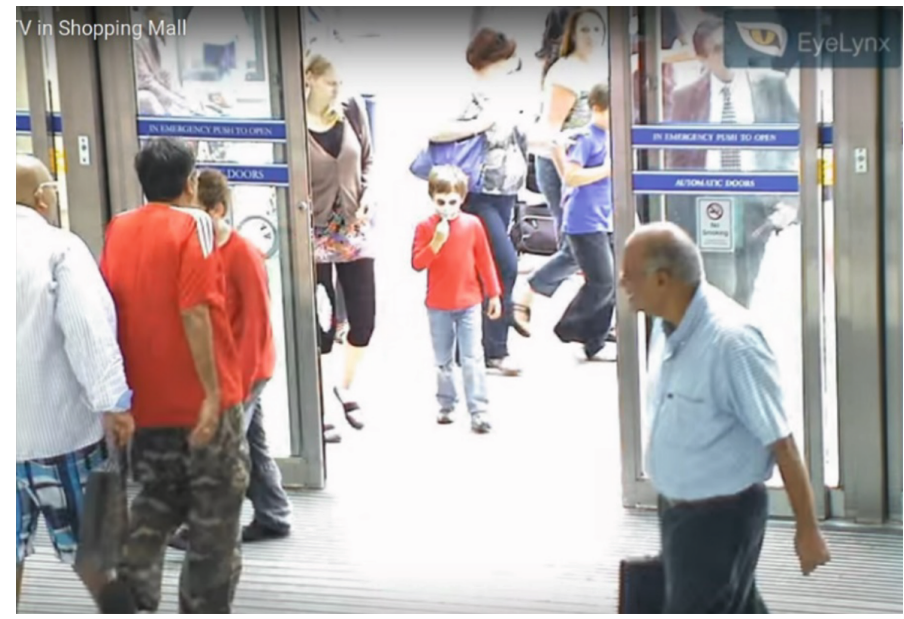

Ilustración 1. Captura de imagen CCTV.

Fuente: YouTube https://www.youtube.com/watch?v=SvldnZ6qMGU (2019). 


\section{Conceptos de redes neuronales y procesamiento de imágenes}

El video digital es una composición de una serie de imágenes desplegadas en una rápida sucesión con frecuencias, comúnmente de 15, 24, 30 y 60 tramas por segundo (Frames Per Second - FPS); entre más tramas tenga el video, más movimiento detallado es capturado o desplegado. (Technopedia, 2019).

Resulta ya bastante común que dispositivos como cámaras digitales o smartphones identifiquen elementos dentro de las imágenes que tratan de obtener: personas, rostros, animales, sonrisas. El campo de la visión artificial y procesamiento de imágenes busca la interpretación del contenido gráfico, utilizando diversas técnicas computacionales, enfocadas al reconocimiento de formas, colores y más allá, al de objetos complejos.

Los sistemas de detección de objetos (Sathyadevan, Balakrishnan y Arya, 2014), actualmente emplean modelos matemáticos basados en arquitecturas de redes neuronales profundas. Estas redes son la evolución de los modelos de redes neuronales concebidos en los años 80 pero que no tuvieron suficiente aceptación debido a la falta de recursos computacionales en ese momento. Recientemente, estas redes han adquirido mucha fuerza y valor tecnológico debido a su capacidad para aprender características determinadas de objetos aprovechando las prestaciones de los computadores de hoy en día. Antes de hablar de las redes neuronales profundas se hará una breve aproximación de las redes neuronales superficiales como como concepto inicial.

Una red neuronal es un modelo conectado (ilustración 2) que trata de imitar el funcionamiento del cerebro humano por medio de algoritmos y funciones matemáticas (D. Svozil, 1997), este modelo tiene una amplia gama de aplicaciones que incluyen predicción de datos e identificación de patrones. Para utilizar una red neuronal es necesario realizar un ajuste de parámetros conocido previamente como entrenamiento. Las redes neuronales están compuestas por capas, estas capas se denominan capas de entrada (input layers), capas ocultas (hidden layers) y capas de salida (output layers). Las capas de entrada reciben los datos que van a alimentar la red neuronal y se transfieren a las capas ocultas que tienen diferentes tipos de operaciones matemáticas que procesan los datos, finalmente las capas de salida poseen generalmente una función de activación que ayuda a determinar el tipo de dato que se está procesando. En el caso de reconocimiento de patrones, estas funciones de activación ayudan a determinar qué clase de objeto se está identificando. Por ejemplo, si es una imagen de un perro, un gato o de un refrigerador.

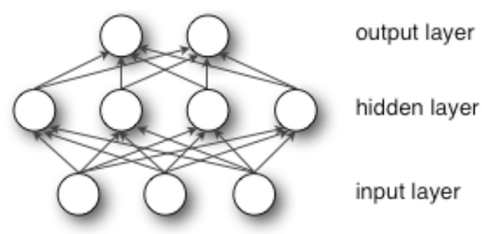

Ilustración 2. Red neuronal.

Fuente: Pathmind (2019). 
Estas funciones matemáticas también se conocen como nodos. En la ilustración 3 se puede apreciar cómo funcionan los nodos de una red neuronal con sus respectivas funciones matemáticas. Estos nodos son la unidad básica fundamental de las redes neuronales y se conocen como perceptrones (Gurney, 2014).

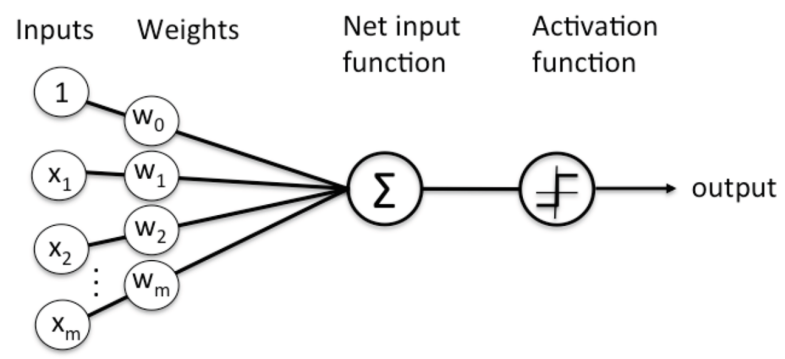

\section{Ilustración 3. Perceptrón.}

Fuente: Pathmind (2019).

- Un perceptrón está compuesto por:

- Entradas (Inputs)

- Pesos (Weights)

- Función de entrada de red (Net input function)

- Función de activación (Activation function)

- Salida (Output)

Las entradas están determinadas por los datos o patrones que se quieren aprender por parte de la red, los pesos son un conjunto de valores que se ajustan por medio del algoritmo de entrenamiento para que la red afiance su aprendizaje de patrones, estos pesos y valores de la entrada se multiplican para posteriormente ser enviados a la función de red que generalmente los suma. Finalmente, esta suma de valores pasa por una función de activación que determina la probabilidad de que los datos de entrada pertenezcan a cierta clase en específica, por ejemplo, la probabilidad de que los datos de entrada pertenezcan a un carro es del $90 \%$.

Matemáticamente se puede definir la salida del perceptrón de la siguiente manera:

$$
\text { salida }=f\left(w_{0}+\sum_{i=1}^{n} x_{i} w_{i}\right)
$$

O mejor

$$
\text { salida }=f\left(w_{0}+w_{1} x_{1}+w_{2} x_{2}+w_{3} x_{3}+\cdots\right)
$$

Dónde $f$ es una función de activación que determina la probabilidad de que esos datos pertenezcan a cierta clase de objeto. 
Una manera de optimizar el proceso de entrenamiento de la red es adecuar los datos de entrada para ahorrar en sí costo computacional y evitar datos redundantes. Por lo tanto, a partir de ahora se nombrarán a los datos de entradas como características de entrada a la red. Si quisiéramos detectar personas en un centro comercial, no basta con introducir muchas fotos para entrenar la red, de hecho, esto podría ocasionar inconvenientes en cuanto al procesamiento. De acuerdo con esto se preparan los datos más relevantes de la imagen que puedan describir a una persona, tales como la altura, forma, color de piel, entre otros. Recordemos que estos datos pueden claramente diferenciarnos de la mayoría de los animales y objetos presentes en una imagen. No obstante, es necesario crear otro algoritmo que pueda extraer estas características para que puedan alimentar a la red neuronal (ilustración 4). Una vez realizada la extracción de características y el adecuado entrenamiento, la red estará en capacidad de poder diferenciar a una persona de cualquier otro objeto dentro de una escena fija o en movimiento.
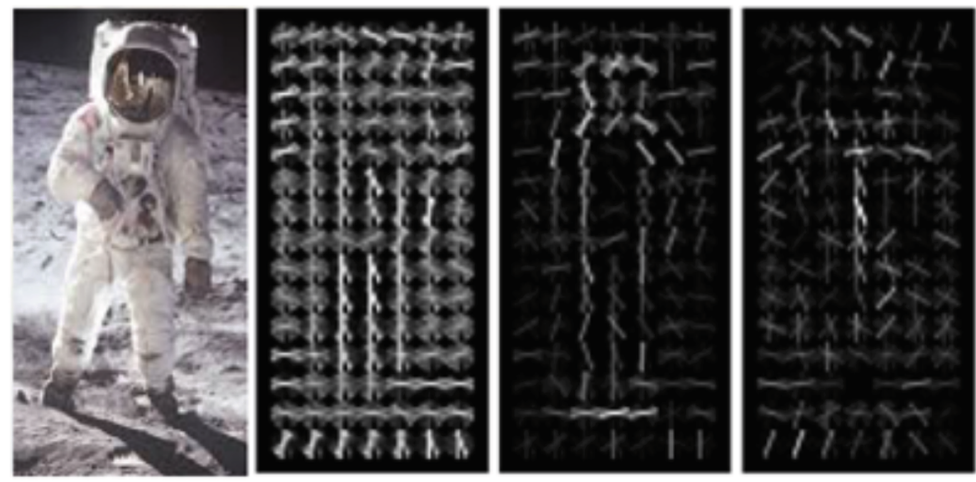

Ilustración 4. Extrayendo características de un astronauta.

Fuente: Intel (2018).

Existe una gran cantidad de algoritmos que extraen características como el presentado en la ilustración 4. Sin embargo, cada uno puede tener ciertas ventajas y desventajas dependiendo de una aplicación específica. Aquí es donde entra el Deep learning o aprendizaje profundo, esta rama utiliza redes neuronales profundas con el propósito de resolver problemas de machine learning. Una red neuronal se considera profunda cuando tiene más de dos capas sin contar la de entrada y salida. La gran ventaja de estas redes neuronales es su capacidad de extraer las características de los objetos por cuenta propia, es decir que, el programador cuenta con un problema menos y es el de elegir el mejor algoritmo que pueda extraer las características relevantes de un objeto para poder entrenar una red y así aprender a identificarlo en cualquier situación.

Este tipo de redes extraen perfectamente las características principales (Yi, Yongliang y Jun, 2019), por ejemplo, del rostro de una mujer u hombre sin necesidad de indicaciones 
específicas con el propósito de identificación de género. El reto en el desarrollo de estas redes radica en definir qué tipo de arquitectura o estilo se va a utilizar para su funcionamiento. Para la detección de objetos, el tipo de red neuronal más utilizado es la red neuronal convolucional (por sus siglas en inglés - CNN). En la Ilustración 5a y 5b se aprecia un esquema general de una arquitectura de red neuronal convolucional (Fukui, 2015) con su respectivo proceso automático de extracción de características.

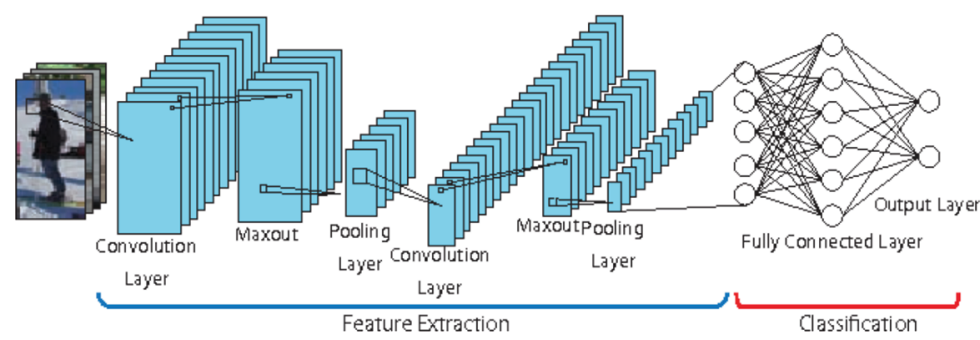

Ilustración 5a. Red neuronal convolucional convencional.

Fuente: (Fukui, 2015), YOLOv3 (Redmon, 2018).

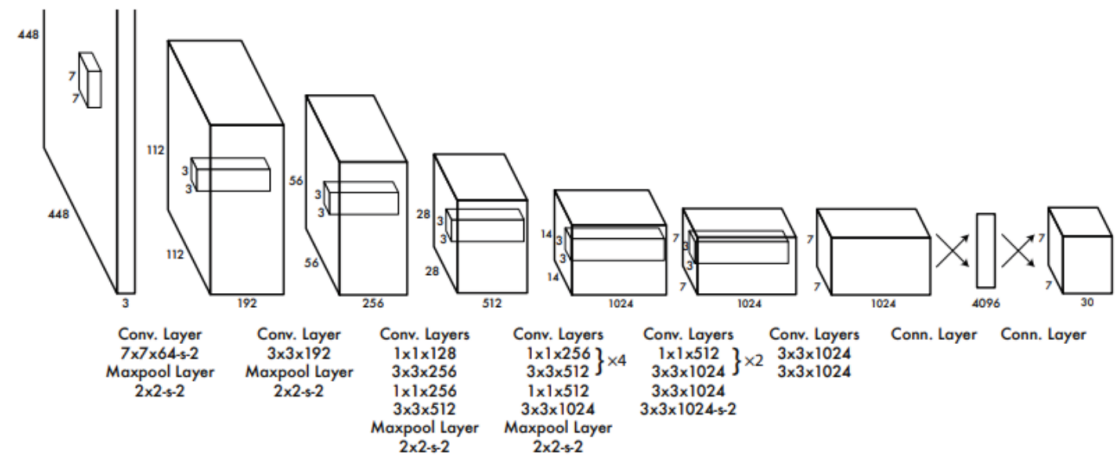

Ilustración 5b. Arquitectura.

Fuente: (Fukui, 2015), YOLOv3 (Redmon, 2018).

En el caso específico de imágenes y video, existe un sistema de detección de objetos conocido como YOLOv3 (Redmon, 2018), esta arquitectura está basada en un modelo modificado de la red neuronal conocida como Darknet. YOLOv3 puede detectar objetos y crear modelos robustos con alta capacidad de generalización (59.7 mAP). Es decir que en el caso de detección de personas posee un alto grado de precisión, además, al ser una red de código abierto cualquier persona con el conocimiento necesario puede utilizar sus 
modelos entrenados para realizar pruebas. No obstante, también se pueden crear modelos propietarios siempre y cuando se cuente con una cantidad suficiente de datos para el respectivo entrenamiento. La arquitectura de YOLOv3 tiende a ser un poco compleja en cuanto al tratamiento matemático y por lo tanto se presenta en forma gráfica en la ilustración $16 b$.

\section{Metodología}

Para la realización de las pruebas de la arquitectura Yolo en sitios públicos; se recurrieron a videos proporcionados por diferentes fuentes como (Denina, 2010) y YouTube. Estos videos presentan situaciones en donde hay un flujo constante de personas a simple vista, posteriormente se escribió un algoritmo que utilizará los videos y el modelo preentrenado de red neuronal con 80 clases como entradas. Cabe anotar que este modelo es suministrado por el equipo creador de Yolo y es de uso público para realizar pruebas. La salida del algoritmo es un video con la detección de las personas y su respectivo etiquetado además de un archivo de base de datos con el número de personas etiquetadas por unidad de tiempo en el video (segundos). El procedimiento general se presenta en la ilustración 6a.

Por otro lado, el algoritmo utilizado junto la red neuronal genera un etiquetado basado en intervalos de confianza que utilizan la probabilidad de que un elemento del video pertenezca a la clase del objeto de interés. Esta probabilidad debe superar un valor umbral establecido previamente, este valor de umbral normalmente es del 50\%.

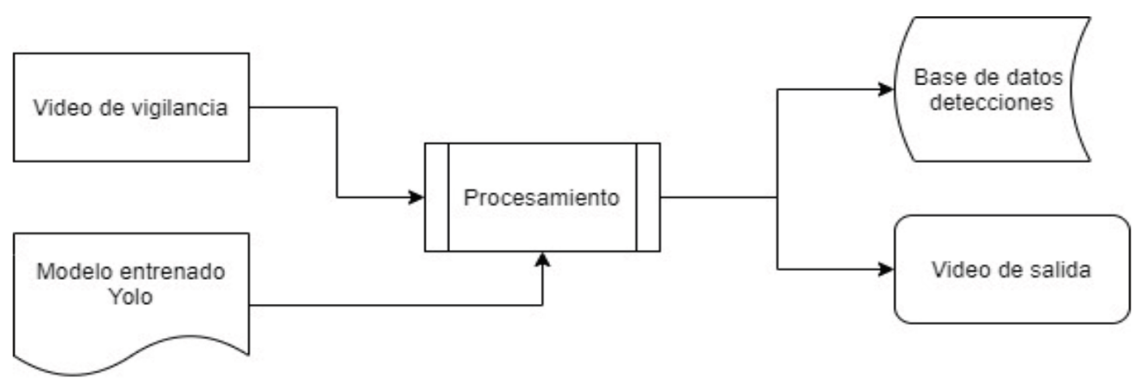

\section{Ilustración 6. a) Proceso principal.}

Fuente: elaboración propia (2019).

La Ilustración $6 \mathrm{~b}$ denota el proceso general definido para el algoritmo que realiza las detecciones teniendo como entrada principal el video de vigilancia. El algoritmo procesa frame a frame el video de la siguiente manera:

1. Dividir cada frame en ventanas de tamaño definido.

2. Procesar cada ventana por medio de la red neuronal para establecer si esa ventana contiene un objeto y si ese objeto pertenece a las clases entrenadas previamente. 
4. Si se ha detectado una clase especifica almacenarla en el archivo de base de datos.

5. Generar el etiquetado gráfico sobre el objeto de interés sobre el frame original.

6. Guardar el nuevo frame dentro de un objeto de video nuevo.

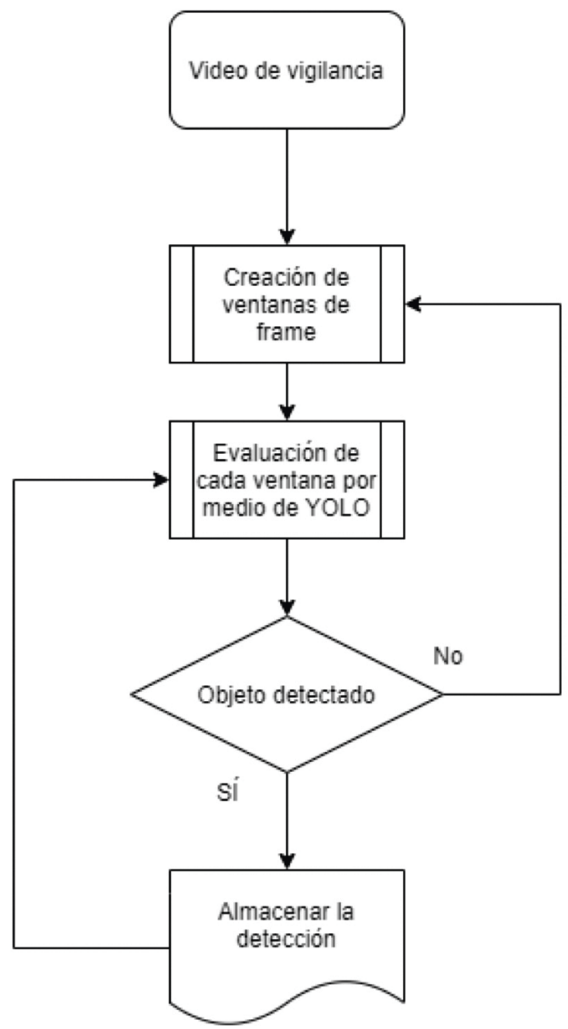

\section{Ilustración 6b. Procesamiento básico de la red neuronal.}

Fuente: elaboración propia (2019).

Una vez obtenidos los diferentes videos procesados se validaron de manera visual teniendo en cuenta que Yolo es un modelo de detección que tiene altos estándares de desempeño respecto a su precisión y tiempo de procesamiento reflejado en la gráfica 2 (Redmon, 2018). 


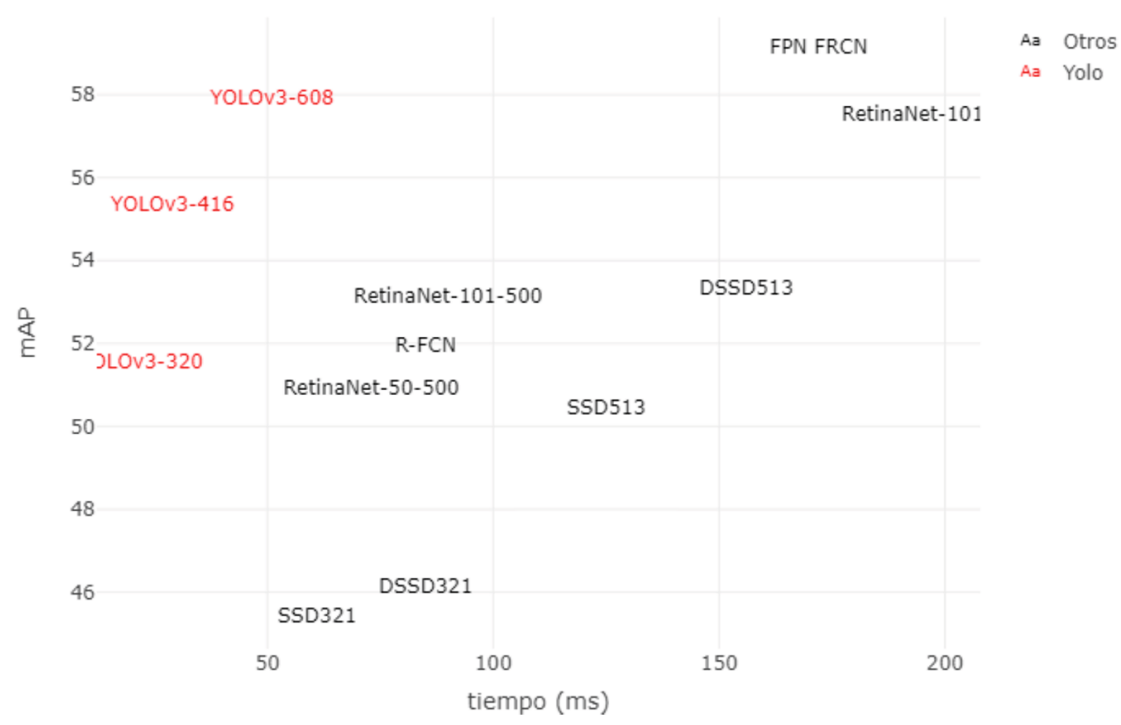

Gráfica 2. Relación tiempo de procesamiento vs mAP entre Yolo y otros modelos

Fuente: elaboración propia (2019).

\section{Pruebas de detección de objetos}

Se realizaron pruebas de detección de personas en cuatro videos generados por (Denina, 2010). Por otro lado, se construyeron bases de datos con la detección de cada uno de los objetos presente en la escena y se construyeron gráficas con la cantidad presente en cada segundo. Esto con el fin de lograr ubicar objetos determinados en algún instante de tiempo sin necesidad de volver a procesar el video. La ilustración 7a presenta una escena tomada del video donde se observa la circulación de personas en un área común. La implementación de YOLOv3 muestra la respectiva detección (ilustración 7b), indicando con una etiqueta el objeto reconocido (person) seguido del nivel de precisión encontrado (ejemplo 0.9). Ampliando a todo el video, la gráfica 3 muestra el número de personas por segundos (Khan, 2016). 


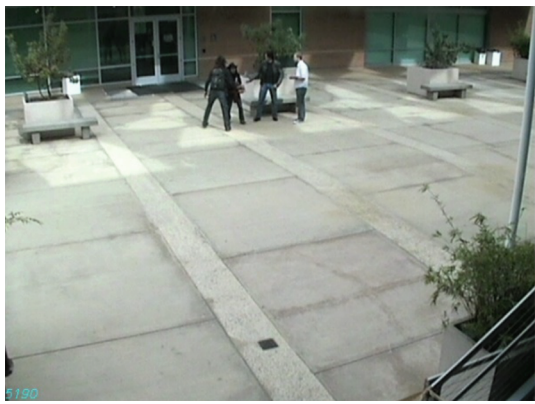

Ilustración 7a. Personas en plaza no procesada.

Fuente: (Denina, 2010).

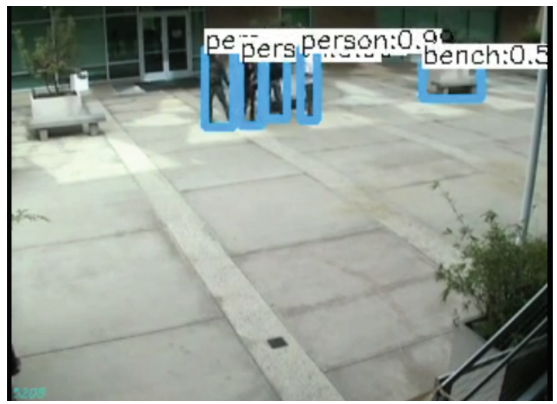

Ilustración $7 \mathrm{~b}$. Con reconocimiento.

Fuente: (Denina, 2010).

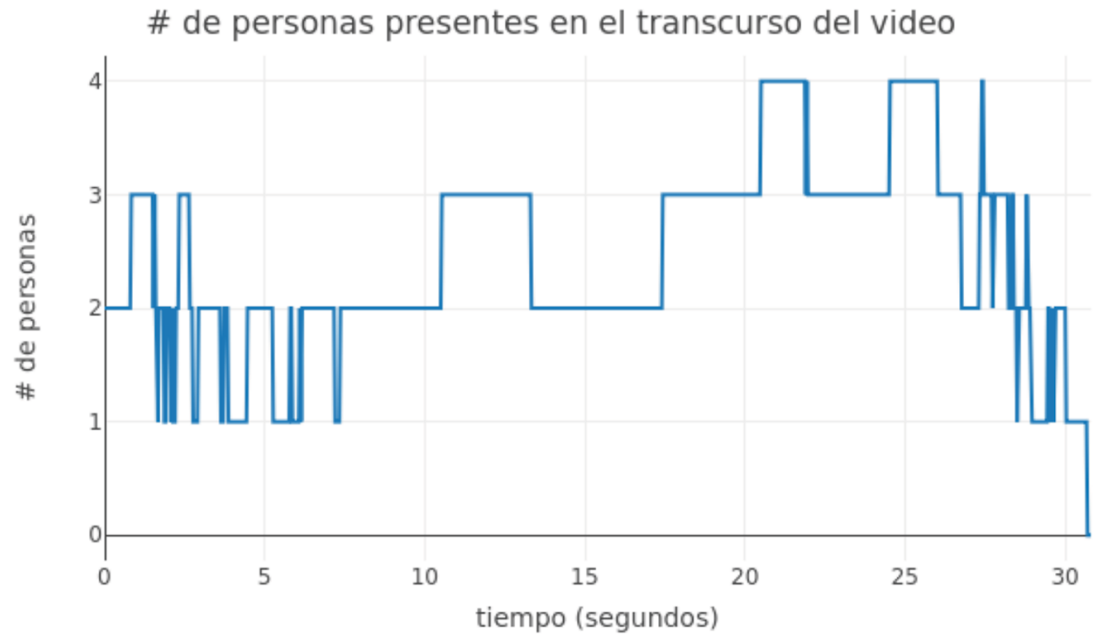

Gráfica 3. Personas en plaza. Línea de tiempo.

Fuente: elaboración propia (2019).

El procesamiento de otra trama se presenta en la ilustración 8 , y el comportamiento del número de personas en el video en la gráfica 4. 


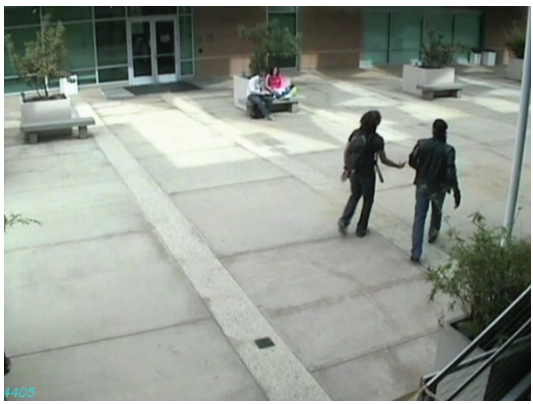

Ilustración 8a. Sin detección.

Fuente: (Denina, 2010).

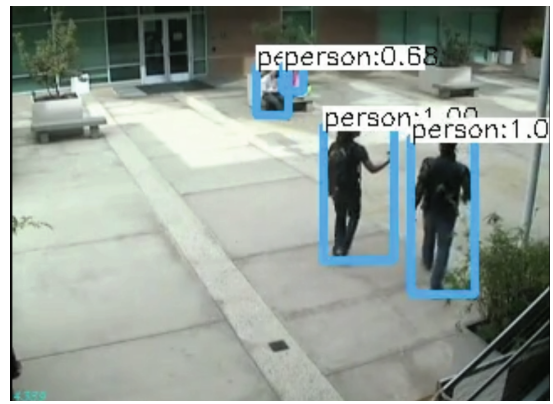

Ilustración 8b. Trama con detección.

Fuente: (Denina, 2010).

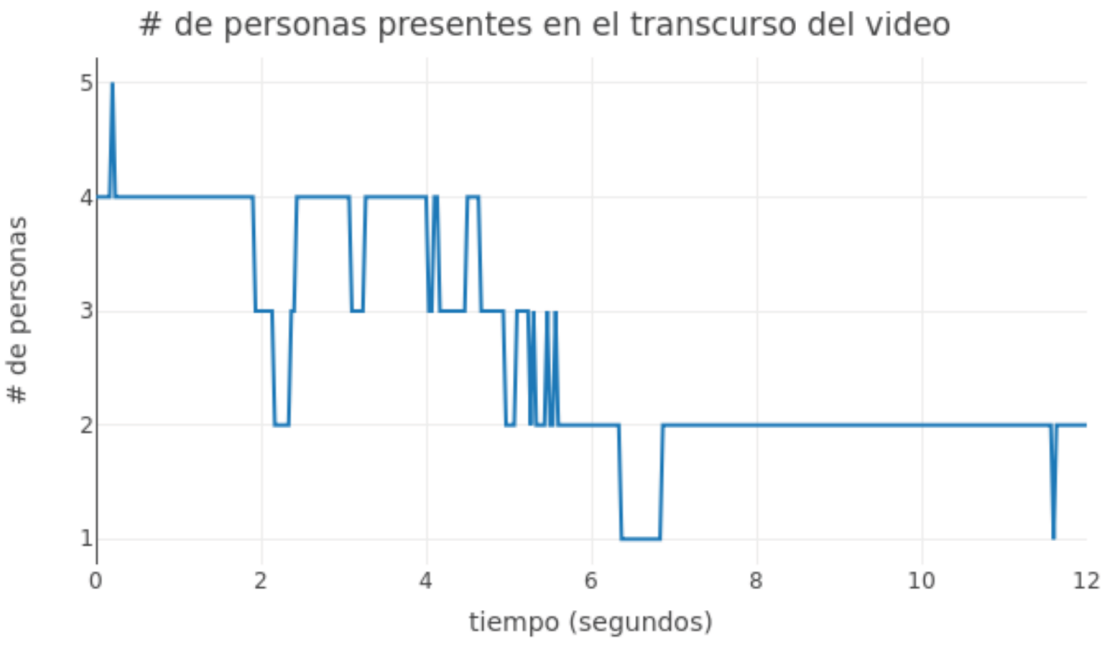

Gráfica 4. . Flujo de personas en el video.

Fuente: elaboración propia (2019).

En la escena 2 (ilustración 9), el nivel de precisión sobre la identificación de personas es del 100\%; la gráfica 5, indica el comportamiento del número de personas durante el tiempo. 


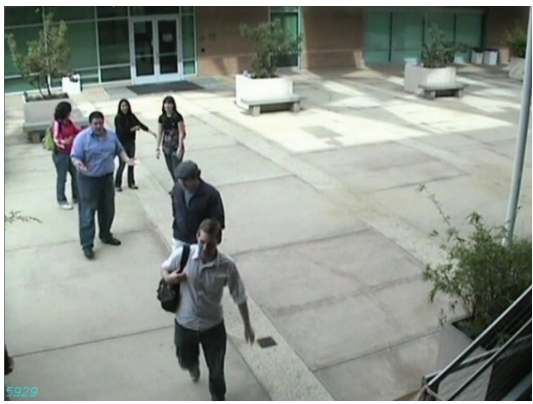

Escena 2. Ilustración $9^{\mathrm{a}}$. Trama $\sin$ detección.

Fuente: (Denina, 2010).

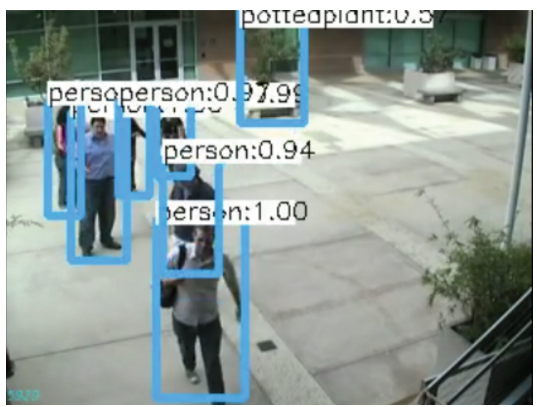

Escena 2. Ilustración 9b. Trama con detección.

Fuente: (Denina, 2010).

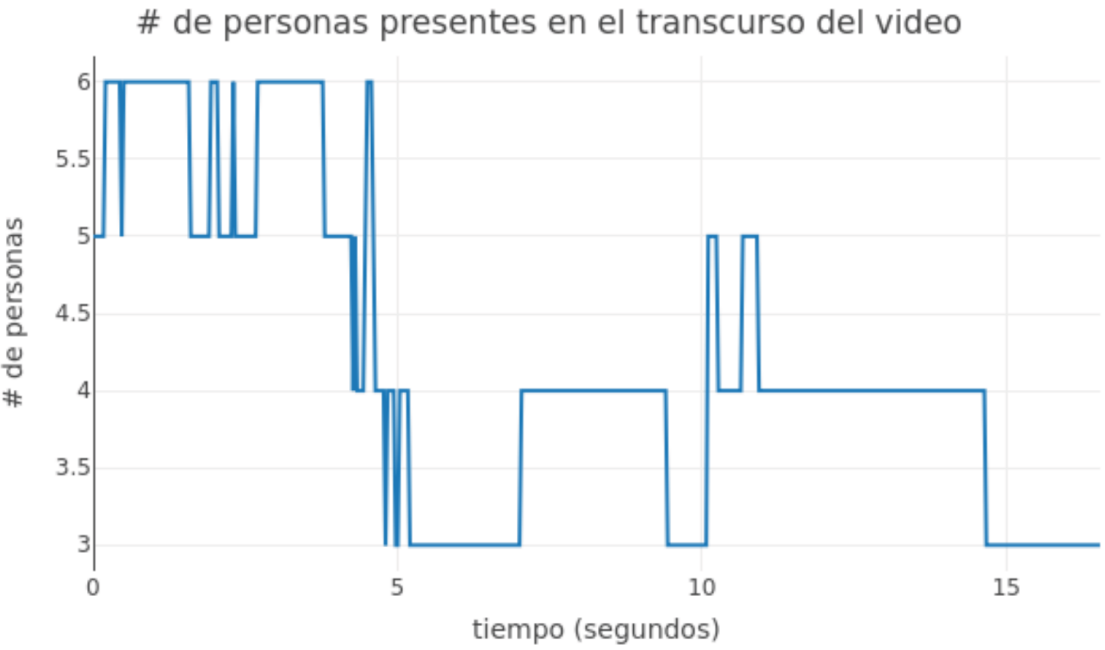

\section{Gráfica 5. Flujo de personas en el video.}

Fuente: elaboración propia (2019).

La tercera escena procesada mostrada en la ilustración 10, indica que el procesamiento no se realiza solo sobre personas, sino sobre cualquier objeto disponible en la base entrenada, identificando plantas y bancas, a diferencia de la gráfica 6 , que sigue contabilizando únicamente el número de personas.

El procesamiento del video en tiempo real presenta el desafío de generar los resultados esperados, al menos, a medida que las tramas se van generando. En este orden de ideas, 
si los videos se encuentran en 24 FPS (tramas por segundo), estas 24 imágenes se deben procesar en máximo 1 segundo.

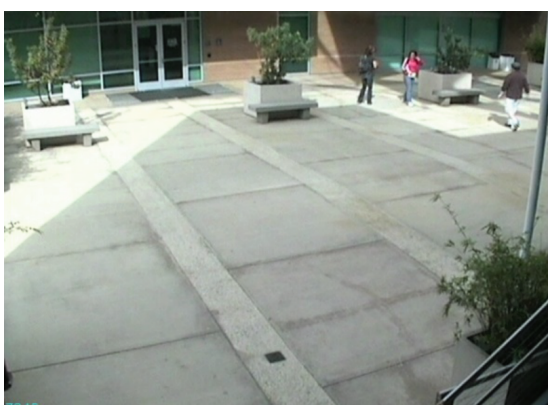

Escena 3. Ilustración 10a. trama sin detección.

Fuente: (Denina, 2010).

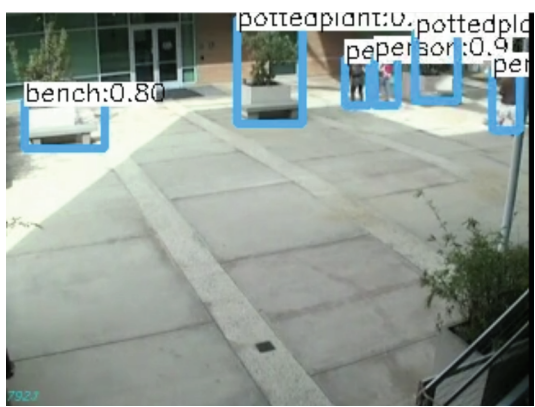

Escena 3. Ilustración 10b. Trama con detección.

Fuente: (Denina, 2010).

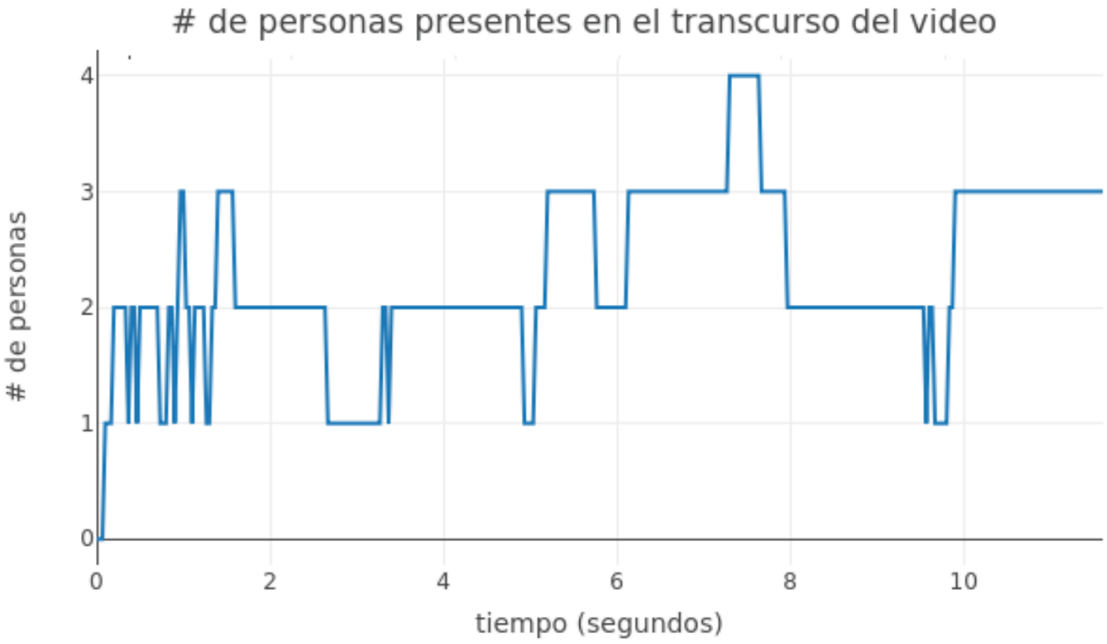

Gráfico 6. Flujo de personas en el video.

Fuente: elaboración propia (2019). 


\section{Resultados y discusión}

Como se había denotado antes, los establecimientos de comercio y particularmente los centros comerciales, tienen una gran afluencia en Colombia. Se puede concluir, que conocer el detalle del número de personas que ingresan o salen del establecimiento en cada momento del día, es un dato de interés y útil para la toma de decisiones desde distintos puntos de vista. Particularmente, desde una empresa de seguridad privada, esta información puede derivar en el uso de ciertos recursos en horas o días específicos, a partir de identificar mayor afluencia de personas, por ejemplo, entre las 3 y 5 de la tarde los últimos viernes de cada mes. Una muestra del procesamiento a partir de CCTV de ilustración 1, asimismo, se tiene en la ilustración 11 y un uso más detallado para reconocimiento de elementos se muestra en la ilustración 12 y 13 .

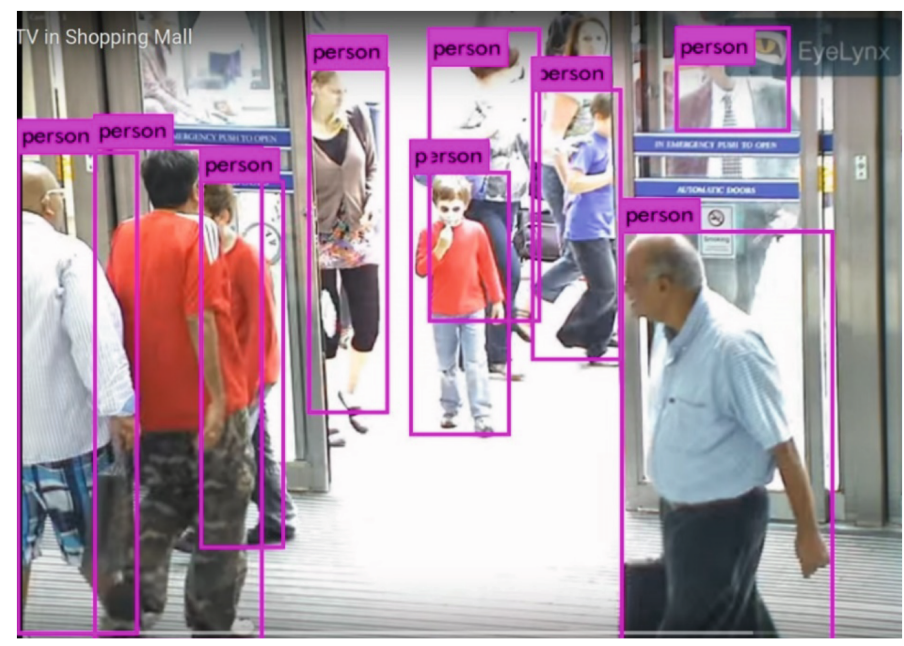

\section{Ilustración 11. Centro comercial. Escena 1.}

Fuente: Recuadros y textos propios (https://www.youtube.com/watch?v=SvldnZ6qMGU (2019).

La revolución en el procesamiento de video y reconocimiento de imágenes que trae industria 4.0 puede ampliar el espectro de la seguridad al de la vigilancia, visto como la posibilidad de equipar con sistemas inteligentes de reconocimiento de personas, elementos sospechosos, incluso con la identificación de aspectos demográficos, vestimenta o calzado específico utilizados por las personas. La recolección de esta información genera valor agregado al concepto de seguridad porque permite obtener detalles en tiempo casi real sobre la afluencia de personas al establecimiento, notar de manera temprana aglomeraciones en sectores específicos, y en consecuencia tomar las medidas necesarias para evitar problemas de seguridad en el sitio. (Ilustración 12). 


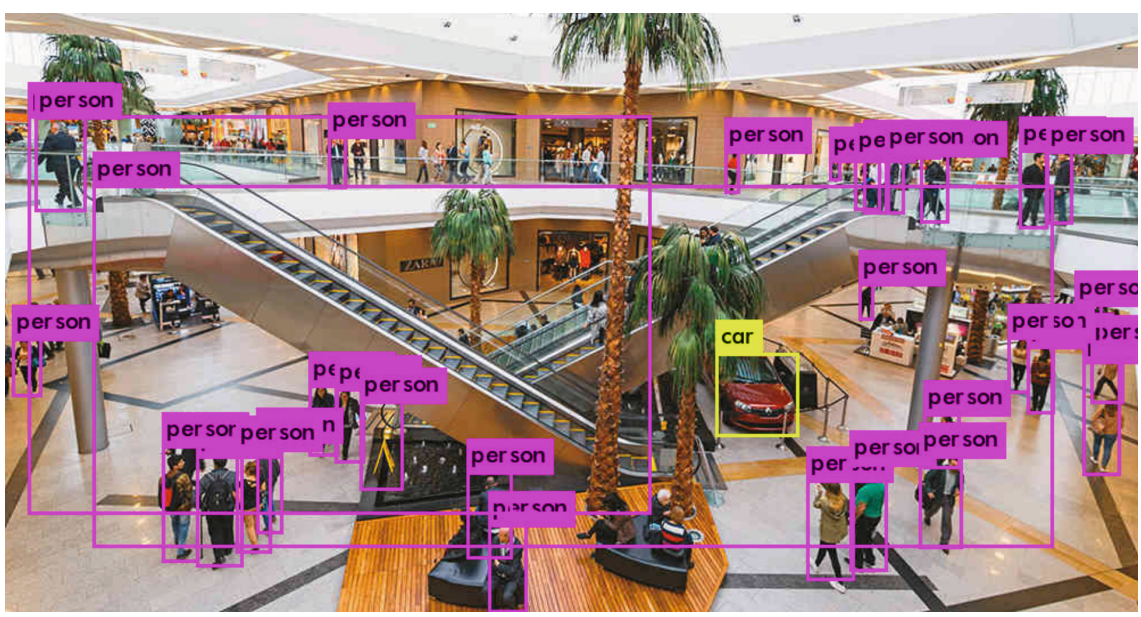

Ilustración 12. C.C Unicentro Bogotá. Recuadros y textos propios. C.C Unicentro Bogotá.

Fuente: Recuadros y textos propios. Revista Semana 2018 (https://www.semana.com/nacion/ articulo/evacuan-centro-comercial-unicentro/555994).

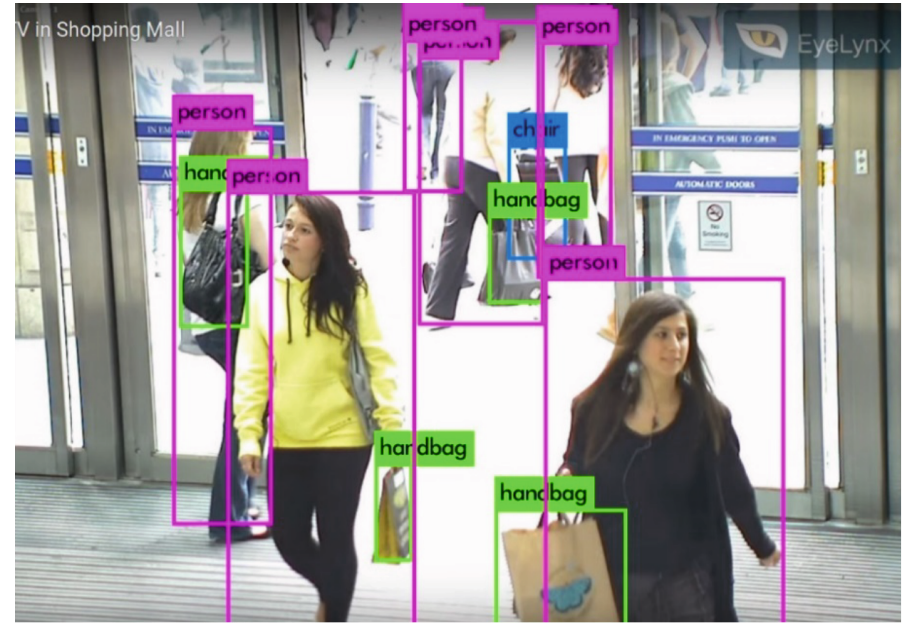

\section{Ilustración 13. Centro Comercial - Escena 2.}

Recuadros y textos propios. (https://www.youtube.com/watch?v=SvldnZ6qMGU (2019). 


\section{Conclusiones}

La posibilidad de que el video procesado se convierta en datos útiles para la toma de decisiones en distintos niveles, proporciona también una ventaja competitiva para los establecimientos de comercio y para las mismas empresas de seguridad, ofertando nuevos servicios complementarios que encajan también en el concepto más amplio de vigilancia. Esta información tiene un amplio potencial de uso, desde aspectos logísticos que permitan optimizar servicios de mantenimiento de zonas, iluminación y servicios conexos, hasta efectos de mercadeo para las tiendas y puntos de acceso de clientes: ¿es de interés si están ingresando más hombres que mujeres, en que horarios, días, por donde circulan dentro del centro comercial?, ¿qué uso y decisiones se pueden tomar con la entrega de esta información de forma oportuna?

Las empresas también se encontrarán con la posibilidad de construir localmente sus sistemas de reconocimiento, hacer uso de servicios en la nube dedicados a visión artificial o desarrollar soluciones mixtas. En todo caso, las decisiones más acertadas deberán asociarse con la visión de negocio, sopesando autonomía, seguridad y costos de operación. Con la accesibilidad a Internet cada vez mayor, más rápida y posiblemente más económicas (como las redes móviles 5G), se tendrán muchas más posibilidades por parte de las organizaciones.

Finalmente, la propuesta de valor más atractiva estará en construir bases de entrenamiento especializadas para distintos fines: identificación de vehículos en detalle, detectar situaciones de emergencia, notar situaciones sospechosas o delictivas, entre otros.

\section{Referencias bibliográficas}

Bouma, H., Baan, J., Burghouts, G. J., Eendebak, P. T., Van Huis, J. R., Dijk, J., y Van Rest, J. H. (2014, October). Automatic detection of suspicious behavior of pickpockets with track-based features in a shopping mall. In Optics and Photonics for Counterterrorism, Crime Fighting, and Defence X; and Optical Materials and Biomaterials in Security and Defence Systems Technology XI (Vol. 9253, p. 92530F). International Society for Optics and Photonics.

Chen, Y., Guo, S., Zhang, B, y Du, K. L. (2013, December). A pedestrian detection and tracking system based on video processing technology. In 2013 Fourth Global Congress on Intelligent Systems (pp. 69-73). IEEE.

D. Svozil, V. K. (1997). Chemometrics and intelligent laboratory systems Introduction to multi-layer feed-forward neural networks.

Fuentes, L. M., y Velastin, S. A. (2004). Tracking-based event detection for CCTV systems. Pattern analysis and applications, 7(4), 356-364.

G. Denina, B. B.-C. (2010). VideoWeb Dataset for Multi-camera Activities and Nonverbal Communication. Distributed Video Sensor Networks (Eds. B. Bhanu, C. Ravishankar, A. Roy-Chowdhury, H. Aghajan, D. Terzopoulos).

George, A. (2018). The benefits of installing CCTV cameras in shopping malls and retail shops. Obtenido de https://medium.com/@abigailgeorge666/the-benefits-ofinstalling-cctv-cameras-in-shopping-malls-and-retail-shops-4e9d1c7c320d 
Gurney, K. (2014). An Introduction to Neural Networks. CRC Press.

H. Fukui, T. Y. (2015). Pedestrian detection based on deep convolutional neural network with ensemble inference network. IEEE Intelligent Vehicles Symposium, Proceedings.

Intel (2018). Intel Software. How Can a Spacecraft Use AI?. Recuperado de: https:// software.intel.com/en-us/blogs/2018/06/06/how-can-a-spacecraft-use-ai

Keval, H. U., y Sasse, M. A. (2008, April). Can we ID from CCTV? Image quality in digital CCTV and face identification performance. In Mobile Multimedial Image Processing, Security, and Applications 2008 (Vol. 6982, p. 69820K). International Society for Optics and Photonics.

Khan, S. (2016). Automatic Detection and Computer Vision Analysis of Flow Dynamics and Social Groups in Pedestrian Crowds.

Lio, V. (2015). Ciudades, Cámaras de seguridad y videovigilancia: estado del arte y perspectivas de investigación. Astrolabio. Nueva Época.

Ministerio de Comercio, Industria y Turismo. (2012). Ley 1581 de 2012. Protección de datos personales. Colombia.

Pathmind (2019). Pathmind. A Beginner's Guide to Neural Networks and Deep Learning. Recuperado de: https://pathmind.com/wiki/neural-network

Pérez, C., y Navarrete, N. (2018). El sector de seguridad y vigilancia privada: evolución reciente y principales retos laborales, regulatorios y de supervisión. Cuadernos de Fedesarrollo. No. 65, 154.

Redmon, J. a. (2018). YOLOv3: An Incremental Improvement. arXiv.

Security Magazine. (2009). Latin American Physical Security Market Growing Rapidly.

Sathyadevan, S., Balakrishnan, A. K., y Arya, S. (2014, August). Identifying moving bodies from CCTV videos using machine learning techniques. In 2014 First International Conference on Networks \& Soft Computing (ICNSC2014) (pp. 151-157). IEEE.

Technopedia. (2019). Digital Video. Obtenido de Technopedia: https://www.techopedia. com/definition/5505/digital-video-dv

Yi, Z., Yongliang, S., y Jun, Z. (2019). An improved tiny-yolov3 pedestrian detection algorithm. Optik, 183, 17-23. 